\title{
Global existence for a quasilinear wave equation outside of star-shaped domains
}

\author{
Hart F. Smith
}

\begin{abstract}
This talk describes joint work with Chris Sogge and Markus Keel, in which we establish a global existence theorem for null-type quasilinear wave equations in three space dimensions, where we impose Dirichlet conditions on a smooth, compact star-shaped obstacle $\mathcal{K} \subset \mathbb{R}^{3}$. The key tool, following Christodoulou [1], is to use the Penrose compactification of Minkowski space. In the case under consideration, this reduces matters to a local existence theorem for a singular obstacle problem. Full details will appear in our joint paper of the same title.
\end{abstract}

We consider a quasilinear system of equations of the form

$$
\left\{\begin{array}{l}
\partial_{t}^{2} u-\Delta u=F\left(u, d u, d^{2} u\right), \quad(t, x) \in \mathbb{R}_{+} \times \mathbb{R}^{3} \backslash \mathcal{K} \\
\left.u(t, \cdot)\right|_{\partial \mathcal{K}}=0 \\
u(0, \cdot)=f, \quad \partial_{t} u(0, \cdot)=g
\end{array}\right.
$$

where $u=\left(u_{1}, \ldots, u_{N}\right)$.

The obstacle $\mathcal{K}$ is assumed to be smooth, compact, and strictly star-shaped with respect to the origin.

The quasilinearity assumption means that $F$, which is assumed to be a smooth function of its arguments, is linear with respect to second order derivatives of $u$, so that

$$
F^{I}\left(u, d u, d^{2} u\right)=G^{I}(u, d u)+\sum_{0 \leq j, k \leq 3} \gamma^{I, j k}(u, d u) \partial_{j} \partial_{k} u^{I}, \quad 1 \leq I \leq N .
$$

We also assume that the nonlinearities satisfy the null condition of Christodoulou [1] and Klainerman [3]. This first of all implies that $F$ vanishes quadratically in its arguments,

$$
F(0,0,0)=0, \quad \text { and } \quad F^{\prime}(0,0,0)=0 .
$$

It also requires that the quadratic terms of $F$ be independent of $u$, so that they take the form

$$
s(d u, d u)+k\left(d u, d^{2} u\right)
$$


where $s$ and $k$ are bilinear forms.

The null condition then can be stated as requiring that the symbols of $s$ and $k$ vanish on null covectors. As shown in [1] and [3], this can equivalently be stated as requiring the semilinear form $s$ to be a linear combination of the basic null forms

$$
q_{0}\left(d u^{J}, d u^{K}\right)=\partial_{0} u^{J} \partial_{0} u^{K}-\sum_{j=1}^{3} \partial_{j} u^{J} \partial_{j} u^{K}
$$

and

$$
q_{i j}\left(d u^{J}, d u^{K}\right)=\partial_{i} u^{J} \partial_{j} u^{K}-\partial_{j} u^{J} \partial_{i} u^{K}, \quad 0 \leq i, j \leq 3,
$$

and the quasilinear form $k$ to be a linear combination of terms of the form

$$
q\left(d u^{J}, d \partial_{j} u^{I}\right), \quad 0 \leq j \leq 3,
$$

where $q$ is a basic null form as above, along with terms of the form

$$
\partial_{j} u^{J}\left(\partial_{t}^{2} u^{I}-\Delta u^{I}\right), \quad 0 \leq j \leq 3 .
$$

Under the null hypothesis, Christodoulou [1] and Klainerman [3] established independently the existence of global solutions to (1) for small initial data $f$ and $g$ in the case that $\mathcal{K}$ is not present. (In the absence of the null condition global existence can fail, as shown by an example of John [2].) Klainerman established this result by exploiting favorable commutation behavior of the wave operator with a certain family of vector fields, in order to establish weighted energy estimates. Together with weighted Sobolev estimates, this establishes sufficient decay of the solution to yield global existence. The technique of Christodoulou was to compactify Minkowski space via the Penrose transform, and to use the fact that null equations behave well under this compactification, to reduce the problem to one of local existence on a curved space, the so called Einstein diamond. Our work follows more closely the method of Christodoulou.

For our work, as in [1], the data $f$ and $g$ for (1) are assumed to be small in appropriate weighted Sobolev spaces. As in [1], let

$$
\|f\|_{H^{m, j}\left(\mathbb{R}^{3}\right)}=\sum_{|\alpha| \leq m}\left(\int_{\mathbb{R}^{3}}\left(1+|x|^{2}\right)^{|\alpha|+j}\left|\partial_{x}^{\alpha} f(x)\right|^{2} d x\right)^{1 / 2} .
$$

In addition, we need to assume that $f$ and $g$ satisfy certain compatibility conditions at the boundary of $\mathcal{K}$. These are induced by the requirement that $\partial_{t}^{k} u$ must vanish on $\partial \mathcal{K}$ for $k$ of whatever order the restriction makes sense. Since $\partial_{t}^{k} u(0, x)$ for $0 \leq k \leq m$ can be expressed in terms of derivatives of $f$ and $g$ of order at most $m$, this implies that the data must satisfy certain nonlinear relations among their derivatives, known as the compatibility conditions of order $m$.

Our main theorem can now be stated as follows.

Theorem 1 Assume that $\mathcal{K}$ and $F\left(u, d u, d^{2} u\right)$ are as above. Assume further that $(f, g) \in C^{\infty}\left(\mathbb{R}^{3} \backslash \mathcal{K}\right)$ satisfy the compatibility conditions to infinite order. Then there exists $\varepsilon_{0}>0$, such that if

$$
\|f\|_{H^{9,8}\left(\mathbb{R}^{3} \backslash \mathcal{K}\right)}+\|g\|_{H^{8,9}\left(\mathbb{R}^{3} \backslash \mathcal{K}\right)}<\varepsilon_{0},
$$


then there is a unique solution $u \in C^{\infty}\left(\mathbb{R}_{+} \times \mathbb{R}^{3} \backslash \mathcal{K}\right)$ of $(1)$. Furthermore, for all $\sigma>0$, there exists $C_{\sigma}<\infty$, such that

$$
|u(t, x)| \leq C_{\sigma}(1+t)^{-1}(1+|t-| x||)^{-1+\sigma} .
$$

We remark that we also establish global existence of solutions $u$ of limited regularity for general data $f$ and $g$ of small norm as above, which are assumed to satisfy the compatibility condition of order 8 . One should be able to replace 9 and 8 respectively by 4 and 3 and let $\sigma=0$, as is the case in [1], but our proof does not show this.

The main tool in proving Theorem 1 is Penrose's conformal compactification of Minkowski space. This is a map $\mathcal{P}: \mathbb{R} \times \mathbb{R}^{3} \rightarrow \mathbb{R} \times S^{3}$, whose image is the set

$$
\left\{(T, X) \in(-\pi, \pi) \times S^{3}:|T|+R<\pi\right\} .
$$

To define the map, let $(R, \omega)$ denote geodesic polar coodinates about the north pole $(1,0,0,0) \in S^{3}$. If $x=r \omega$ denotes polar coodinates in $\mathbb{R}^{3}$, then the Penrose map preserves the angular variable $\omega$, while the remaining variables are related by

$$
\begin{aligned}
& R=\arctan (t+r)-\arctan (t-r), \\
& T=\arctan (t+r)+\arctan (t-r) .
\end{aligned}
$$

Under this map the pushforward of the Minkowski metric $d t^{2}-d x^{2}$ is related to the Lorentz metric $d T^{2}-d X^{2}$ on $(-\pi, \pi) \times S^{3}$ as follows,

$$
d T^{2}-d X^{2}=\Omega^{2}\left(d t^{2}-d x^{2}\right)
$$

where the conformal factor $\Omega$ is given by the formula

$$
\Omega=\cos T+\cos R=\frac{2}{\left(1+(t+r)^{2}\right)^{1 / 2}\left(1+(t-r)^{2}\right)^{1 / 2}},
$$

with $(T, R)$ and $(t, r)$ being identified as above.

Continuing, let

$$
\square=\partial_{T}^{2}-\Delta_{S^{3}}
$$

be the D'Alembertian coming from the standard Laplace-Beltrami operator $\Delta_{S^{3}}$ on $S^{3}$. If we let $\tilde{\square}$ denote the D'Alembertian on $\mathbb{R}^{1+3}$, then the two wave operators are related as follows,

$$
\square+1=\Omega^{-3} \tilde{\square} \Omega,
$$

with the additive constant 1 arising because of the non-zero scalar curvature of $S^{3}$. Equivalently,

$$
\tilde{\square} \tilde{u}=\tilde{F} \Longleftrightarrow(\square+1) u=F \text { with } \tilde{u}=\Omega u \text { and } F=\Omega^{-3} \tilde{F} .
$$

Consequently, if

$$
\mathcal{K}_{*}=\mathcal{P}(\mathbb{R} \times \mathcal{K})
$$


is the pushforward of the obstacle $\mathcal{K}$, then proving existence of global solutions to the system (1) for small Cauchy data reduces to showing that we can find solutions for time $T \in(-\pi, \pi)$ for small Cauchy data to the system

$$
\left\{\begin{array}{l}
(\square+1) u=\Omega^{-3} F\left(\Omega u, d(\Omega u), d^{2}(\Omega u)\right), \quad(T, X) \in(-\pi, \pi) \times S^{3} \backslash \mathcal{K}_{*} \\
u(T, X)=0, \quad(T, X) \in \partial \mathcal{K}_{*} .
\end{array}\right.
$$

The first issue to be dealt with is to show that the nonlinearity is actually a smooth expression in $u$ and its derivatives in the $(T, X)$ variables, which is not immediate due to the term $\Omega^{-3}$, which is singular at $T+R=\pi$. It is the quadratic terms in $F$ that thus present a possible obstruction to global solvability. Christodoulou [1] showed that the transformed quadratic terms extend smoothly to $\mathbb{R} \times S^{3}$ precisely when the null condition is verified (see [1], p. 277-278), from which global existence for the obstacle-free case follows by local existence for smooth quasilinear equations on $S^{3}$.

This proof breaks down for our problem due to the fact that the transformed obstacle $\mathcal{K}_{*}$ is a time-dependent obstacle which collapses to a point as $T \rightarrow \pi$. Indeed, for some constant $C$, we have that for $0 \leq T<\pi$,

$$
C^{-1}(\pi-T)^{2} \leq \operatorname{dist}(X, \mathbf{1}) \leq C(\pi-T)^{2}, \quad \text { if } \quad(T, X) \in \partial \mathcal{K}_{*},
$$

with 1 denoting the north pole on $S^{3}$.

The start of the proof for the obstacle case is that the energy inequality holds in the above setting provided that $\mathcal{K}$ is star-shaped. To state this, we let

$$
Y=(-\pi, \pi) \times S^{3} \backslash \mathcal{K}_{*} .
$$

Theorem 2 Suppose that $\mathcal{K} \subset \mathbb{R}^{3}$ is star-shaped with respect to the origin. Suppose also that $u \in C^{2}$ and $u(T, X)=0$ if $(T, X) \in \partial Y$, and let

$$
F=\square u \text {. }
$$

Then, for $0 \leq T<\pi$,

$$
\left\|u^{\prime}(T, \cdot)\right\|_{L^{2}\left(Y_{T}\right)} \leq\left\|u^{\prime}(0, \cdot)\right\|_{L^{2}\left(Y_{0}\right)}+\int_{0}^{T}\|F(S, \cdot)\|_{L^{2}\left(Y_{S}\right)} d S .
$$

Here, $u^{\prime}$ denotes the $(T, X)$ gradient of $u$. The proof of Theorem 2 follows from the fact that the energy flux across $\partial Y$ has the correct sign if $\mathcal{K}$ is star-shaped. This proof still holds under small perturbations of the metric, and hence will hold for the perturbed equation on $Y$.

To obtain $C^{2}$ bounds on solutions $u$, which are neccessary to close the loop so we may apply the energy inequality, we need to control the $L^{2}$ norm of higher derivatives of $u$. Since the obstacle $\mathcal{K}_{*}$ is time-dependent, the $T$ derivatives of $u$ do not satisfy the Dirichlet condition, and so we cannot use the standard trick of controlling $\partial_{T}^{k} u$. The one vector field at our disposal which does preserve Dirichlet conditions is the pushforward of $\partial_{t}$. Since this field vanishes as $(\pi-T)^{2}$, we consequently end up controlling only powers of the weighted derivatives

$$
Z=(\pi-T)^{2} \partial_{T, X}
$$

With this notation we establish the following. 
Theorem 3 Assume that the system (1) satisfies the null condition. Then there exists $\delta_{0}>0$, so that if

$$
\|f\|_{H^{9}\left(Y_{0}\right)}+\|g\|_{H^{8}\left(Y_{0}\right)} \leq \delta_{0},
$$

then the pushforward of the system (1) via the Penrose transform has a solution $u$ on $Y$ with Cauchy data $(f, g)$ which verifies

$$
\begin{aligned}
\sup _{0 \leq T<\pi} \sum_{|\alpha| \leq 8}\left(\left\|Z^{\alpha} u^{\prime}(T, \cdot)\right\|_{L^{2}\left(Y_{T}\right)}+\right. & \left.\left\|Z^{\alpha} u(T, \cdot)\right\|_{L^{6}\left(Y_{T}\right)}\right) \\
& +\sup _{0 \leq T<\pi}(\pi-T)^{\sigma} \sum_{|\alpha| \leq 5}\left\|Z^{\alpha} u(T, \cdot)\right\|_{L^{\infty}\left(Y_{T}\right)}<\infty
\end{aligned}
$$

for all $\sigma>0$.

We remark that the pointwise bounds on $u$ in the theorem do not follow directly from the stated $L^{2}$ and $L^{6}$ bounds on $u$. To establish the pointwise bounds, we resort to the equation, and use $L^{1} \rightarrow L^{\infty}$ bounds for the non-perturbed wave equation on $(-\pi, \pi) \times S^{3}$. The proof also exploits the fact that the coefficients of the push forward of a null quasilinear equation, in addition to being smooth in the $(T, X)$ variables, also vanish suitably at the tip of the diamond $T=\pi, R=0$. For example, the coefficients $\gamma^{I, j k}(T, X ; u, d u)$ of the second order derivatives in $T$ and $X$ for the pushed forward equation satisfy the bounds

$$
\begin{aligned}
& \left|Z^{\alpha} \gamma^{I, j k}\right| \leq(\pi-T)^{4} \sum_{|\gamma| \leq|\alpha|}\left|Z^{\gamma} u^{\prime}\right|+(\pi-T)^{3} \sum_{|\gamma| \leq|\alpha|}\left|Z^{\gamma} u\right|+ \\
& \quad \sum_{\left|\gamma_{1}\right|+\left|\gamma_{2}\right| \leq|\alpha|}\left((\pi-T)^{2}\left|Z^{\gamma_{1}} u\right|+(\pi-T)^{4}\left|Z^{\gamma_{1}} u^{\prime}\right|\right)\left((\pi-T)^{2}\left|Z^{\gamma_{2}} u\right|+(\pi-T)^{4}\left|Z^{\gamma_{2}} u^{\prime}\right|\right)
\end{aligned}
$$

assuming that

$$
(\pi-T)^{2} \sum_{|\gamma| \leq 1+|\alpha| / 2}\left|Z^{\gamma} u\right| \leq B
$$

where $B$ is a fixed constant.

These bounds also show that the mild blowup of $u$ as $T \rightarrow \pi$ does not lead to blowup of the coefficients $\gamma^{I, j k}$.

\section{References}

[1] D. Christodoulou: Global solutions of nonlinear hyperbolic equations for small initial data, Comm. Pure Appl. Math. 39 (1986), 267-282.

[2] F. John: Nonlinear wave equations, formation of singularities, University Lecture Series, American Mathematical Society, 1990.

[3] S. Klainerman: The null condition and global existence to nonlinear wave equations, Nonlinear systems of partial differential equations in applied mathematics, Part 1 (Santa Fe, N.M., 1984), 293-326, Lectures in Appl. Math., 23, Amer. Math. Soc., Providence, R.I., 1986. 
Department of Mathematics,

UNIVERSITY OF WASHINGTON,

Seattle, WA 98195

hart@math . washington. edu 